\section{Willingness of tobacco smokers to contribute financially towards cessation resources}

To the Editor: Exposure to tobacco smoke accounts for approximately 9\% (95\% confidence interval 8.1 - 10.2) of all South African (SA) deaths. ${ }^{[1]}$ Seventeen percent of SA adults are self-reported active smokers and an alarming 32\% of pregnant women were found to be active smokers on urine cotinine testing. ${ }^{[2,3]}$ Despite the publication in 2013 of the South African Tobacco Smoking Cessation Clinical Practice Guideline, ${ }^{[4]}$ tobacco smoking cessation programmes in the public health sector remain scarce.

We conducted a cross-sectional survey in 2010 of 100 adult smokers at Tygerberg Academic Hospital in Cape Town to determine the rate of nicotine dependence using the Fagerström questionnaire and alcohol co-dependence using the CAGE questionnaire. ${ }^{[5,6]}$ We also assessed willingness to contribute financially to smoking cessation resources. The Human Research Ethics Committee of Stellenbosch University approved the study (N10/05/179).

The majority of subjects were identified by smoking behaviour outside entrance number 2, the entrance to Tygerberg Children's

Table 1. Demographic, tobacco and alcohol dependence characteristics $(N=100)$

\begin{tabular}{|c|c|}
\hline \multicolumn{2}{|l|}{ Demographic } \\
\hline Age (yr), median (IQR) & $34(27-43)$ \\
\hline Female, $n(\%)$ & $57(57)$ \\
\hline \multicolumn{2}{|l|}{ Education, $n(\%)$} \\
\hline$<$ Grade 8 & $21(21)$ \\
\hline Grade $8-12$ & $66(66)$ \\
\hline Any tertiary education & $13(13)$ \\
\hline Employed & $58(58)$ \\
\hline \multicolumn{2}{|l|}{ Home environment } \\
\hline Number of people in house, median (IQR) & $5(3-6)$ \\
\hline $\begin{array}{l}\text { Number of smokers in house, median } \\
\text { (IQR) }\end{array}$ & $2(1-3)$ \\
\hline $\begin{array}{l}\text { Number of children (<13 years) in house, } \\
\text { median (IQR) }\end{array}$ & $1.5(0.5-2)$ \\
\hline \multicolumn{2}{|l|}{$\begin{array}{l}\text { Tobacco dependence by Fagerström score, } \\
n(\%)\end{array}$} \\
\hline High & $17(17)$ \\
\hline Medium & $49(49)$ \\
\hline Low & $34(34)$ \\
\hline \multicolumn{2}{|l|}{ Alcohol use, $n(\%)$} \\
\hline Drinks alcohol & $43(43)$ \\
\hline Others in house drink alcohol & $43(43)$ \\
\hline $\begin{array}{l}\text { Alcohol dependent (by CAGE } \\
\text { questionnaire) }\end{array}$ & $22(22)$ \\
\hline
\end{tabular}

Hospital. The median age of participants was 34 years (interquartile range (IQR) 27 - 43 years). Two-thirds were parents or caregivers accompanying child patients to the hospital (Table 1). Despite $54 \%$ having reported smoking fewer than 10 cigarettes per day, two-thirds had medium to high nicotine dependence and $22 \%$ had alcohol co-dependence (Table 1). Ninety-three percent understood that smoking was dangerous both to themselves and to others. Sixty-four percent had been advised by a healthcare worker to quit and $72 \%$ had unsuccessfully attempted to quit at least once. Eightyone percent (52/64) of those advised to quit and 67\% (24/36) not advised had made at least one attempt to do so. Sixty-eight percent of smokers were willing to pay the equivalent of their current cigarette expenditure for cessation resources. Those willing to pay tended to have a higher rate of attempting cessation compared with those not willing to pay ( $82 \%$ v. $72 \%, p=0.09$ ). The majority spent from less than ZAR10 to ZAR25 per day on tobacco products and only $9 \%$ spent more than ZAR25 per day. The mean annual expenditure on tobacco products was ZAR6 000 per smoker.

The high rate of medium to high nicotine dependence and the frequent unsuccessful attempts to quit in this sample indicate the need for both professional counselling and pharmacotherapy to assist smoking cessation in this population. These smokers were financially invested in the process and willing to contribute financially to cessation resources, an unexplored opportunity for smokers and the public health sector to share the financial responsibility of smoking cessation programmes in SA.

Acknowledgement. We gratefully acknowledge the late Prof. Chris Bollinger's advice on this study.

\section{Karthik Rao}

Johns Hopkins University School of Medicine, Baltimore, MD, USA

\section{Amy L Slogrove}

Department of Paediatrics and Child Health, Stellenbosch University, Cape Town, South Africa

\section{Louise Cooke}

Department of Paediatrics, University of Cape Town, South Africa

\section{Mark F Cotton}

Division of Paediatric Infectious Diseases and Family Clinical Research Unit (FAM-CRU), Stellenbosch University, Cape Town, South Africa mcot@sun.ac.za

1. Institute for Health Metrics and Evaluation. Deaths and DALYs due to smoking and second han smoke exposure: South Africa. Global Burden of Disease (GBD) Results Tool 2015. http://ghdx. healthdata.org/gbd-results-tool? params=querytool-permalink/lad6db1837cded0235571b1374a600b (accessed 9 October 2016)

2. Reddy P, Zuma K, Shisana O, Kim J, Sewpaul R. Prevalence of tobacco use among adults in South Africa: Results from the first South African National Health and Nutrition Examination Survey $S$ A Med J 2015;105(8):648-655. http://dx.doi.org/10.7196/SAMInew.7932

3. Vanker A, Barnett W, Brittain $\mathrm{K}$, et al. Antenatal and early life tobacco smoke exposure in an African birth cohort .

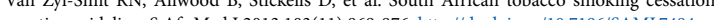

Deaher Dependence: A revision of the Fagerström Tolerance Questionnaire. Br J Addict 1991;86(9):1119-1127. 6. Ewing JA. Detecting alcoholism: The CAGE questionnaire. JAMA 1984;252(14):1905-1907. 